\title{
The Transition from Regular to Irregular Motions, Explained as Travel on Riemann Surfaces
}

\author{
F Calogero ${ }^{1,2}$, D Gómez-Ullate ${ }^{3}, \mathbf{P}$ M Santini ${ }^{1,2}$, and M \\ Sommacal $^{4}$ \\ 1 Dipartimento di Fisica, Università di Roma "La Sapienza", Roma, Italy. \\ 2 Istituto Nazionale di Fisica Nucleare, Sezione di Roma, Italy. \\ ${ }^{3}$ Dep. Matemàtica Aplicada I, Universitat Politècnica de Catalunya, ETSEIB, \\ Av. Diagonal 647, 08028 Barcelona, Spain. \\ 3 SISSA, Triste, Italy. \\ E-mail: francesco.calogero@roma1.infn.it, david.gomez-ullate@upc.edu, \\ paolo.santini@roma1.infn.it, sommacal@sissa.it
}

\begin{abstract}
We introduce and discuss a simple Hamiltonian dynamical system, interpretable as a 3-body problem in the (complex) plane and providing the prototype of a mechanism explaining the transition from regular to irregular motions as travel on Riemann surfaces. The interest of this phenomenology - illustrating the onset in a deterministic context of irregular motions - is underlined by its generality, suggesting its eventual relevance to understand natural phenomena and experimental investigations. Here only some of our main findings are reported, without detailing their proofs: a more complete presentation will be published elsewhere.
\end{abstract}

PACS numbers: 05.45-a, 02.30.Hq, 02.30.Ik. 


\section{Introduction}

Purpose and scope of this paper is to introduce and discuss a simple Hamiltonian dynamical system describing the motion of 3 particles in the (complex) plane. This 3 -body problem is the prototype of a class of models [1-3] that feature a transition from very simple (even isochronous) to quite complicated motions characterized by a sensitive dependence both on the initial data and the parameters ("coupling constants") of the model. This transition can be explained as travel on Riemann surfaces. The interest of this phenomenology - illustrating the onset in a deterministic context of irregular motions - is underlined by its generality [1,3], suggesting its eventual relevance to understand natural phenomena and experimental investigations. The novelty of the model treated herein is that it allows a quite explicit mathematical treatment. Here only some of our main findings are reported, without detailing their proofs: a more complete presentation will be published elsewhere [4].

The idea that the integrable or nonintegrable character of a dynamical system is closely related to the analytic structure of its solutions as functions of the independent variable ("time", but considered as a complex variable) goes back to such eminent mathematicians as Carl Jacobi, Sophia Kowalewskaya, Henri Poincaré, Paul Painlevé and his school. Some of us heard illuminating discussions of this notion by Martin Kruskal, whose main ideas - a synthetic if overly terse rendition of which might be the statement that integrability is compatible with the presence of multivaluedness but only provided this is not excessive - can be gleaned from some papers written by himself and some of his collaborators [5], or by others who performed theoretical and numerical investigations motivated by his ideas [6]. The results presented below constitute progress along this line of thinking. For a more detailed analysis we refer the interested reader to the papers where more complete versions are presented of our findings [4].

\section{Results}

The model we introduce and discuss in this paper is characterized by the following equations of motion:

$$
\dot{z}_{n}=-\mathrm{i} \omega z_{n}+\frac{g_{n+2}}{z_{n}-z_{n+1}}+\frac{g_{n+1}}{z_{n}-z_{n+2}} .
$$

Notation: here and hereafter indices such as $n, m$ range from 1 to 3 and are defined $\bmod (3)$; superimposed dots indicate differentiations with respect to the real independent time variable $t$; the dependent variables $z_{n} \equiv z_{n}(t)$ are complex, and indicate the positions of 3 point "particles" moving in the complex $z$-plane; $\mathrm{i} \equiv \sqrt{-1}$ is the imaginary unit; the parameter $\omega$ is positive, and it sets the time scale via the basic period

$$
T=\frac{\pi}{\omega}
$$

the 3 quantities $g_{n}$ are arbitrary coupling constants, but in this paper we restrict consideration to the case in which they are all real and moreover satisfy the "semisymmetrical" restriction

$$
g_{1}=g_{2}=g, \quad g_{3}=f,
$$


entailing that the two particles with labels 1 and 2 are equal, while particle 3 is different. More special cases are the "fully symmetrical", or "integrable", one characterized by the equality of all 3 coupling constants,

$$
f=g, \quad g_{1}=g_{2}=g_{3}=g,
$$

and the "two-body" one, with only one nonvanishing coupling constant,

$$
g_{1}=g_{2}=g=0, g_{3}=f \neq 0 .
$$

In this latter case clearly

$$
z_{3}(t)=z_{3}(0) \exp (-\mathrm{i} \omega t)
$$

and the remaining two-body problem is easily solvable,

$$
\begin{aligned}
z_{s}(t)=\exp (-\mathrm{i} \omega t)\left[\frac{1}{2}\left[z_{1}(0)+z_{2}(0)\right]\right. \\
\left.-(-)^{s}\left\{\frac{1}{4}\left[z_{1}(0)-z_{2}(0)\right]^{2}+f \frac{\exp (2 \mathrm{i} \omega t)-1}{2 \mathrm{i} \omega}\right\}^{1 / 2}\right], \quad s=1,2 .
\end{aligned}
$$

The justification for labelling the fully symmetrical case (4) as "integrable" will be clear from the following (or see Section 2.3.4.1 of [3]). The treatment of the more general case with 3 different coupling constants $g_{n}$ is outlined in [4].

Note that the equations of motion (11) are of "Archimedian", rather than "Newtonian", type, inasmuch as they imply that the "velocities", rather than the "accelerations", are determined by the "forces". These equations of motion are Hamiltonian, indeed they follow in the standard manner from the Hamiltonian function

$$
H(\mathbf{z}, \mathbf{p})=\sum_{n=1}^{3}\left[-\mathrm{i} \omega z_{n} p_{n}+g_{n} \frac{p_{n+1}-p_{n+2}}{z_{n+1}-z_{n+2}}\right] .
$$

And they can be reformulated [4] as, still Hamiltonian, real (and covariant, even rotation-invariant) equations describing the motion of three point particles in the (real) horizontal plane.

The following qualitative analysis (confirmed by our quantitative findings, see below) is useful to get a first idea of the nature of the motions entailed by our model. For large values of (the modulus of) $z_{n}$ the "two-body forces" represented by the last two terms in the right-hand side of (10) become negligible with respect to the "onebody (linear) force" represented by the first term, hence in this regime $\dot{z}_{n} \approx-i \omega z_{n}$ entailing $z_{n}(t) \approx$ const $\exp (-i \omega t)$. One thereby infers that, when a particle strays far away from the origin in the complex $z$-plane, it tends to rotate (clockwise, with period $2 T$ ) on a circle: hence the first qualitative conclusion that all motions are confined. Secondly, the two-body forces cause a singularity whenever there is a collision of two (or all three) of the particles, and become dominant whenever two particles get very close to each other, namely in the case of near misses. But if the three particles move aperiodically in a confined region (near the origin) of the complex z-plane, an infinity of near misses shall indeed occur. And since the outcome of a near miss is generally quite different (whenever the two particles involved in it are different) depending on which side the particles slide past each other - and this, especially in the case of very close near misses, depends sensitively on the initial data of the trajectories under consideration - we see here a mechanism causing a sensitive dependence of the 
time evolution on its initial data. This suggests that our model (1), in spite of its simplicity, might also support quite complicated motions, possibly even displaying an "unpredictable" evolution in spite of its deterministic character. This hunch is confirmed by the results reported below.

To investigate the dynamics of our "physical" model (1) it is convenient to introduce an "auxiliary" model, obtained from it via the following change of dependent and independent variables:

$$
z_{n}(t)=\exp (-\mathrm{i} \omega t) \zeta_{n}(\tau), \quad \tau(t)=\frac{\exp (2 \mathrm{i} \omega t)-1}{2 \mathrm{i} \omega} .
$$

Note that initially the coordinates $z_{n}$ and $\zeta_{n}$ coincide:

$$
z_{n}(0)=\zeta_{n}(0) \text {. }
$$

The equations of motion of the auxiliary model follow immediately from (11) via (7) (or, even more directly, by noting that, for $\omega=0, \tau=t$ and $z_{n}(t)=\zeta_{n}(\tau)$ ):

$$
\zeta_{n}^{\prime}=\frac{g_{n+2}}{\zeta_{n}-\zeta_{n+1}}+\frac{g_{n+1}}{\zeta_{n}-\zeta_{n+2}} .
$$

Here of course the appended prime denotes differentiation with respect to the (complex) variable $\tau$.

The definition of $\tau(t)$ implies that as the (real) time variable $t$ evolves onwards from $t=0$, the complex variable $\tau$ travels round and round, making a full tour (counterclockwise) in every time interval $T$, on the circle $C$ the diameter of which, of length $d=1 / \omega$, lies on the imaginary axis in the complex $\tau$-plane, with one end at the origin, $\tau=0$, and the other at $\tau=\mathrm{i} / \omega$ (draw this circle!). Hence these relations, (17), entail that if $\zeta_{n}(\tau)$ is holomorphic as a function of the complex variable $\tau$ in the closed disk $D$ encircled by the circle $C$, the corresponding function $z_{n}(t)$ is periodic in the real variable $t$ with period $2 T$ (indeed antiperiodic with period $T$ ):

$$
z_{n}(t+T)=-z_{n}(t), \quad z_{n}(t+2 T)=z_{n}(t) .
$$

But it is easy to prove [4] that the solution $\zeta_{n}(\tau)$ of (9) is holomorphic (at least) in the circular disk $D_{0}$ centered at the origin of the complex $\tau$-plane and having the radius

$$
r=\frac{\left(\min _{n, m=1,2,3 ; m \neq n}\left|\zeta_{n}(0)-\zeta_{m}(0)\right|\right)^{2}}{128 \max _{n=1,2,3}\left|g_{n}\right|} .
$$

One may therefore conclude that our physical system (10) is isochronous with period $2 T$, see (2). Indeed an isochronous system is characterized by the property to possess one or more open sectors of its phase space, each having of course full dimensionality, such that all motions in each of them are completely periodic with the same fixed period (the periods may be different in these different sectors of phase space, but must be fixed, i e. independent of the initial data, within each of these sectors): and in our case clearly (at least) all the motions characterized by initial data $z_{n}(0)$ such that

$$
\min _{n, m=1,2,3 ; m \neq n}\left|z_{n}(0)-z_{m}(0)\right|>16 \sqrt{\frac{\max _{n=1,2,3}\left|g_{n}\right|}{2 \omega}}
$$

are completely periodic with period $2 T$, see (10), since this inequality, implying (via (8) and (11) $r>d$, entails that $\zeta_{n}(\tau)$ is holomorphic (at least) in a disk $D_{0}$ that includes, in the complex $\tau$-plane, the disk $D$. 
This argument is a first demonstration of the usefulness of the "trick" (17), associating the auxiliary system (9) to our physical system (11). More generally, this relationship (7) allows to infer the main characteristics of the time evolution of the solutions $z_{n}(t)$ of our physical system (11) from the analyticity properties of the corresponding solutions $\zeta_{n}(\tau)$ of the auxiliary system (9): indeed the evolution of $z_{n}(t)$ as the time $t$ increases from the initial value $t=0$ is generally related via (7) to the values taken by $\zeta_{n}(\tau)$ when $\tau$ rotates (counterclockwise, with period $T$ ) on the circle $C$ in the complex $\tau$-plane and correspondingly $\zeta_{n}(\tau)$ travels on the Riemann surface associated to its analytic structure as a function of the complex variable $\tau$. Suppose for instance that the only singularities of $\zeta_{n}(\tau)$ in the finite part of the complex $\tau$-plane are square-root branch points, as it is indeed the case for our model (11) at least for a range of values of the ratio of the coupling constants $f$ and $g$, see (3) and below. $\$$ Then the isochronous regime corresponds to initial data such that the corresponding solution $\zeta_{n}(\tau)$ has no branch points inside the circle $C$ on the main sheet of its Riemann surface (i. e. that characterized by the initial data). Moreover, if there is a finite (nonvanishing) number of branch points inside the circle $C$ on the main sheet of the Riemann surface of $\zeta_{n}(\tau)$, and a finite number of branch points inside the circle $C$ on all the sheets that are accessed by traveling on the Riemann surface round and round on the circle $C$, then clearly the corresponding solution $z_{n}(t)$ is still a completely periodic function of the time $t$, but now its period is a finite integer multiple $j T$ of the basic period $T$, the value of $j$ depending of course on the number of sheets that get visited along this travel before returning to the main sheet. Hence, in particular, whenever the total number $q$ of (square-root) branch points of the solution $\zeta_{n}(\tau)$ of the auxiliary problem (9) is finite, the corresponding solution $z_{n}(t)$ of our physical model (1) is completely periodic, although possibly with a very large period (if $q$ is very large) the value of which may depend, possibly quite sensitively, on the initial data. On the other hand if the number of (square-root) branch points possessed by the generic solution $\zeta_{n}(\tau)$ is infinite, and the Riemann surface associated with the function $\zeta_{n}(\tau)$ has an infinite number of sheets (as it can happen in our case, see below), then it is possible that, as $\tau$ goes round and round on the circle $C$, the corresponding value of $\zeta_{n}(\tau)$ travels on this Riemann surface without ever returning to its main sheet, entailing that the time evolution of the corresponding function $z_{n}(t)$ is aperiodic, and that it depends sensitively on the initial data inasmuch as these data characterize the positions of the branch points hence the structure of the Riemann surface.

This terse analysis entails an important distinction among all these (square-root) branch points: the "active" branch-points are those located inside the circle $C$ on sheets of the Riemann surface accessed - when starting from the main sheet - by traveling round and round on that circle, so that they do affect the subsequent sequence of sheets that get visited; while the "inactive" branch points are, of course, those that fall outside the circle $C$, as well as those that are located inside the circle $C$ but on sheets of the Riemann surface that do not get visited while traveling round and round on that circle (starting from the main sheet) and that therefore do not influence the time-evolution of the corresponding solution of our physical system (11). This

$\ddagger$ The nature of these singularities is generally independent from the particular solution under consideration and can be easily ascertained [4] via local analyses à la Painlevé of the generic solution of the equations of motion (9), while the number and especially the locations of these singularities depend on the specific solution under consideration and their identification requires a more detailed knowledge than can be obtained by a local analysis à la Painlevé. 
distinction is of course influenced by the initial data of the problem, that characterize the initial pattern of branch points; clearly it is not just a "local" characteristic of each branch point depending only on its position (for instance, inside or outside the circle $C$ ): it depends on the overall structure of the Riemann surface, for instance if there is no branch point on its main sheet - that containing the point of departure of the travel round and round on the circle $C$ - then clearly all the other branch points are inactive, irrespective of their location.

Let us also emphasize that, whenever an active branch point is quite close to the circle $C$, it corresponds to a near miss involving two particles of our physical model (11), at which these two particles scatter against each other almost at right angles (corresponding to the square-root nature of the branch point). The difference between the cases in which such a branch point falls just inside respectively just outside the circle $C$ corresponds to a near miss in which the two particles slide past each other on one side respectively on the other (see figure 1), and this makes a substantial difference as regards the subsequent evolution of our 3-body system (unless the two particles are equal). The closer the near miss, the more significant this effect is, and
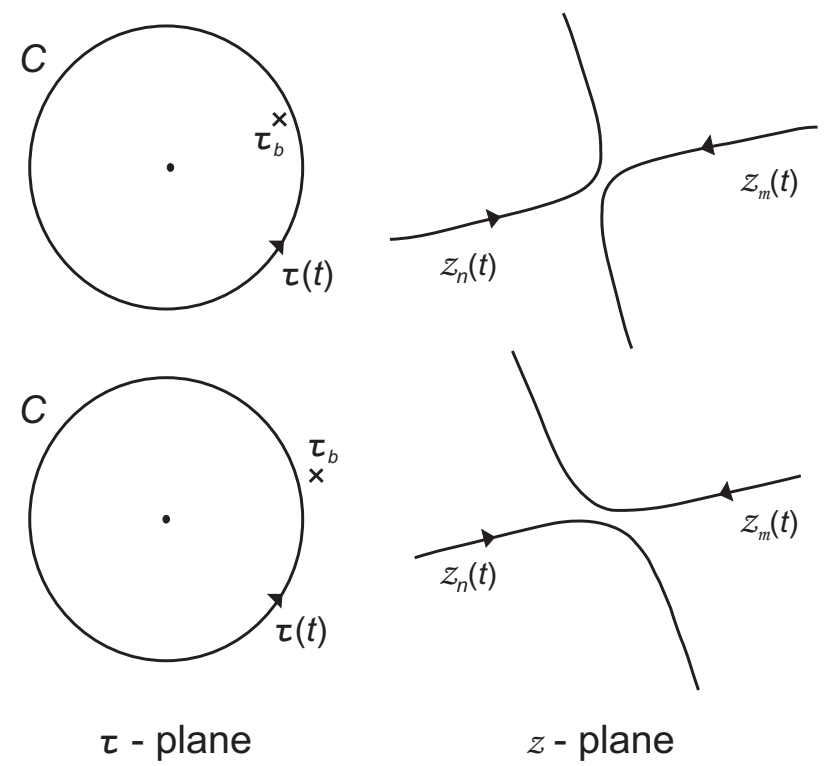

Figure 1. Scattering of two bodies in the three-body problem (1) corresponding to a near miss. The two outcomes originate from two sets of initial data close to each other such that a square-root branch point $\tau_{b}$ falls on different sides of the circle $C$.

the more sensitive it is on the initial data, a tiny change of which can move the relevant branch point from one side to the other of the circumference of the circle $C$ and correspondingly drastically affect the outcome of the near miss. This is the mechanism that accounts for the fact that, when the initial data are in certain sectors of their phase space (of course quite different from that characterized by the inequalities (12)), the resulting motion of the physical 3-body problem (1) is aperiodic, indeed nontrivially so: in such cases (as we show below) the aperiodicity is indeed associated with the coming into play of an infinite number of (square-root) branch points of 
the corresponding solution of the auxiliary problem (9) and correspondingly with an infinite number of near misses experienced by the particles throughout their time evolution, this phenomenology being clearly characterized by a sensitive dependence on the initial data.

This mechanism to explain the transition from regular to irregular motions and in particular from an isochronous regime to one featuring unpredictable aspects - was already discussed [2] in the context of certain many-body models somewhat analogous to that studied herein. But those treatments were limited to providing a qualitative analysis such as that presented above and to ascertaining its congruence with numerical solutions of these models. The interest of the simpler model introduced and discussed herein is to allow a detailed, quantitative understanding of this phenomenology. This is based on the following explicit solution of our model (1), obtained [4] via the auxiliary problem (9):

$$
\begin{gathered}
z_{s}(t)=Z \exp (-\mathrm{i} \omega t)-\frac{1}{2}\left(\frac{f+8 g}{6 \mathrm{i} \omega}\right)^{1 / 2}[1+\eta \exp (-2 \mathrm{i} \omega t)]^{1 / 2} . \\
\cdot\left\{[\check{w}(t)]^{1 / 2}-(-)^{s}[12 \mu-3 \check{w}(t)]^{1 / 2}\right\}, \quad s=1,2, \\
z_{3}(t)=Z \exp (-\mathrm{i} \omega t)+\left(\frac{f+8 g}{6 \mathrm{i} \omega}\right)^{1 / 2}[1+\eta \exp (-2 \mathrm{i} \omega t)]^{1 / 2}[\check{w}(t)]^{1 / 2} .
\end{gathered}
$$

Here the function $\check{w}(t)$ is defined via the relation

$$
\check{w}(t)=w[\xi(t)],
$$

with

$$
\xi(t)=R[\eta+\exp (2 \mathrm{i} \omega t)]=\bar{\xi}+R \exp (2 \mathrm{i} \omega t),
$$

and $w(\xi)$ implicitly defined by the nondifferential equation

$$
(w-1)^{\mu-1} w^{-\mu}=\xi .
$$

The parameter $\mu$ is defined in terms of the coupling constants $g$ and $f$, see (3), as follows:

$$
\mu=\frac{f+2 g}{f+8 g}
$$

and in (13)-(15) the three constants $Z, R$, and $\eta$ (or $\bar{\xi}$ ) are defined in terms of the 3 initial data $z_{n}(0)$ as follows:

$$
\begin{aligned}
& Z=\frac{z_{1}(0)+z_{2}(0)+z_{3}(0)}{3} \\
& R=\frac{3(f+8 g)}{2 \mathrm{i} \omega\left[2 z_{3}(0)-z_{1}(0)-z_{2}(0)\right]^{2}}\left[1-\frac{1}{\check{w}(0)}\right]^{\mu-1}, \\
& \bar{\xi}=R \eta \\
& \eta=\frac{\mathrm{i} \omega\left\{\left[z_{1}(0)-z_{2}(0)\right]^{2}+\left[z_{2}(0)-z_{3}(0)\right]^{2}+\left[z_{3}(0)-z_{1}(0)\right]^{2}\right\}}{3(f+2 g)}-1, \\
& \check{w}(0)=\frac{2 \mu\left[2 z_{3}(0)-z_{1}(0)-z_{2}(0)\right]^{2}}{\left[z_{1}(0)-z_{2}(0)\right]^{2}+\left[z_{2}(0)-z_{3}(0)\right]^{2}+\left[z_{3}(0)-z_{1}(0)\right]^{2}} .
\end{aligned}
$$


Note that the constant $Z$ is the initial value of the center of mass of the system, and indeed the first term in the right-hand side of the solution (13) represents the motion of the center of mass of the system: just a circular motion around the origin, with a constant velocity entailing a period $2 T$. Since the rest of the motion is independent of the behavior of the center of mass, in the study of this model attention can be restricted without significant loss of generality to the case when the center of mass does not move, $Z=0$.

The nontrivial aspects of the motion are encoded in the time evolution of the function $\check{w}(t)$, see (13) and (14): let us emphasize in this connection that the dependent variable $w(\xi)$ is that solution of the nondifferential equation (16) uniquely identified by continuity, as the time $t$ unfolds, hence as the variable $\xi \equiv \xi(t)$ goes round and round, in the complex $\xi$-plane, on the circle $\Xi$ with center $\bar{\xi}$ and radius $|R|$ (see (15)), from the initial datum assigned at $t=0$,

$$
w[\xi(0)]=w(\bar{\xi}+R)=\check{w}(0),
$$

see (18e). This specification of the initial value $\check{w}(0)$ is relevant, because generally the nondifferential equation (16) has more than a single solution, in fact possibly an infinity of solutions, see below.

It is clear from (13) that the time evolution of the solution $z_{n}(t)$ of our model (1) is mainly determined by the time evolution of the function $\check{w}(t)$. Indeed,

(i) The factor $[\eta \exp (-2 \mathrm{i} \omega t)-1]^{1 / 2}$ displays a quite simple time evolution, periodic with period $T$ if $|\eta|<1$ and antiperiodic with period $T$ hence periodic with period $2 T$ if $|\eta|>1$.

(ii) If $\check{w}(t)$ is periodic with period $\check{T}$, its square $\operatorname{root}[\check{w}(t)]^{1 / 2}$, appearing in the righthand side of the solution formulas (13), is clearly as well periodic with period $\check{T}$ or antiperiodic with period $\breve{T}$ hence periodic with period $2 \check{T}$ depending whether the closed trajectory of $\check{w}(t)$ in the complex $\check{w}$-plane does not or does enclose the (branch) point $\check{w}=0$.

(iii) Likewise the square root $[12 \mu-3 \check{w}(t)]^{1 / 2}$ (see [13ad) is also periodic with period $\check{T}$ or antiperiodic with period $\check{T}$ hence periodic with period $2 \check{T}$ depending whether the closed trajectory of $\check{w}(t)$ in the complex $\check{w}$-plane does not or does enclose the (branch) point $\check{w}=4 \mu$ (but note that a change of sign of this square root only entails an exchange between the two equal particles 1 and 2).

(iv) In conclusion one sees that - provided one considers particles 1 and 2 as indistinguishable - then, if the time evolution of $\check{w}(t)$ is periodic with period $\check{T}, \check{w}(t+\check{T})=\check{w}(t)$, the physical motion of the 3 particles $z_{n}(t)$ is also completely periodic either with the same period $\check{T}$ or with period $2 \check{T}$, provided $\check{T}$ is an integer multiple of $T$;

(v) Finally, if the motion of $\breve{w}(t)$ is not periodic then clearly the functions $z_{n}(t)$ are also not periodic.

Hereafter we only discuss the time evolution of the function $\check{w}(t)$; actually, as explained below, in this paper we limit our consideration to discussing the motion of a generic solution $\tilde{w}(t)=w[\xi(t)]$ of the nondifferential equation (16). Moreover, we consider only generic solutions of (10), namely those characterized by initial data that exclude one of the following special outcomes:

(a) $\check{w}(t)$ takes, at some (real) time $t_{a}$, the value $\check{w}\left(t_{a}\right)=4 \mu$ entailing a pair collision of the 2 equal particles occurring at this time, $z_{1}\left(t_{a}\right)=z_{2}\left(t_{a}\right)$. 
(b) $\check{w}(t)$ takes, at some (real) time $t_{b}$, the value $\check{w}\left(t_{b}\right)=\mu$ entailing a pair collision of the different particle with one of the 2 equal particles occurring at this time, $z_{1}\left(t_{b}\right)=z_{3}\left(t_{b}\right)$ or $z_{2}\left(t_{b}\right)=z_{3}\left(t_{b}\right)$.

(c) The constant $\eta$ has unit modulus, $|\eta|=1$, i. e. $\eta=\exp \left(2 \mathrm{i} \omega t_{c}\right)$ with $t_{c}$ real (and of course defined $\bmod T$ ) which entails a triple collision of the 3 particles occurring at the time $t_{c}, z_{1}\left(t_{c}\right)=z_{2}\left(t_{c}\right)=z_{3}\left(t_{c}\right)$.

(c') $\check{w}(t)$ vanishes at some (real) time $t_{c}, \check{w}\left(t_{c}\right)=0$ (but, as our notation suggests, this case (c') is just a subcase of (c), although this is not immediately obvious from (13a) but requires using also (15) and (16)).

The initial data that give rise to solutions having one of these singularities form a set of null measure. It can be easily seen that these singular solutions $z_{n}(t)$ of our physical problem (11) correspond via (7) to special solutions $\zeta_{n}(\tau)$ of our auxiliary problem (9) possessing a branch point that sits exactly on the circle $C$ in the complex $\tau$-plane: more precisely,

(a) a square-root branch point featured by $\zeta_{1}(\tau)$ and $\zeta_{2}(\tau)$ but not by $\zeta_{3}(\tau)$,

(b) a square-root branch point featured by all 3 functions $\zeta_{n}(\tau)$,

(c) a branch point featured by all 3 functions $\zeta_{n}(\tau)$ the nature of which depends on the parameter $\mu$.

As mentioned above, in this paper we confine our treatment to discussing the time evolution of a generic root $\tilde{w}(t)$ of the nondifferential equation (16) with (15), and in particular to identifying for which initial data its time evolution is periodic, and in such a case what the period is. Remarkably we find out that, for (arbitrarily) given initial data, all these roots have at most three different periods (one of which might be infinite, signifying an aperiodic motion); periods which we are able to determine explicitly (although the relevant formulas have some nontrivial, even "chaotic", aspects, in a sense that is made explicit below). The question of identifying, among all the roots $\tilde{w}_{j}(t)$ of this nondifferential equation (16), the "physical" one $\check{w}(t)$ i. e. the one that evolves from the initial datum (18e), and in particular of specifying the character of its time evolution among the (at most 3) alternatives discussed below, is a technically demanding job the solution of which shall be reported in [4]. Let us re-emphasize that the time evolution of $\tilde{w}(t) \equiv w[\xi(t)]$ coincides with the evolution of a generic root $w(\xi)$ of (16) as the independent variable $\xi$ travels (making a complete counterclockwise tour in the complex $\xi$-plane in every time interval $T$ ) on the circle $\Xi$ with center $\bar{\xi}$ and radius $|R|$, see (15), and correspondingly the dependent variable $w(\xi)$ travels on its Riemann surface. Note that this Riemann surface is completely defined by the single parameter $\mu$, see (17) and (16), while the circle $\Xi$ is defined by the initial data of the problem, see (15) with (18).

What therefore remains to be discussed is the analytic structure of the multivalued function $w(\xi)$ defined implicitly by the nondifferential equation (16) or, equivalently but more directly, the time dependence of the corresponding function $\tilde{w}(t) \equiv \tilde{w}[\xi(t)]$. To begin with, we consider the case in which the parameter $\mu$ is rational,

$$
\mu=\frac{p}{q},
$$

with $p$ and $q$ coprime integers and $q$ positive, $q>0$. The extension of the results to the case of irrational $\mu$ is made subsequently; although, to avoid repetitions, we present below some results in a manner already appropriate to include also the more general case with $\mu$ real. 
In the rational case (20) the nondifferential equation that determines the "dependent variable" $\tilde{w}(t)$ in terms of the "independent variable" $t$ becomes polynomial, and takes one of the following 3 forms depending on the value of the parameter $\mu$, see (20):

$$
\begin{array}{ll}
(\tilde{w}-1)^{p-q}=[\bar{\xi}+R \exp (2 \mathrm{i} \omega t)]^{q} \tilde{w}^{p}, & \text { if } \mu>1, \\
{[\bar{\xi}+R \exp (2 \mathrm{i} \omega t)]^{q}(\tilde{w}-1)^{q-p} \tilde{w}^{p}=1,} & \text { if } 0<\mu<1, \\
{[\bar{\xi}+R \exp (2 \mathrm{i} \omega t)]^{q}(\tilde{w}-1)^{q+|p|}=\tilde{w}^{|p|,}} & \text { if } \mu<0 .
\end{array}
$$

The above expressions are polynomials (in the dependent variable $\tilde{w}$ ) of degree $J$ :

$$
J= \begin{cases}p, & \text { if } \mu>1 ; \\ q, & \text { if } 0<\mu<1 ; \\ q+|p|, & \text { if } \mu<0 .\end{cases}
$$

As for the boundaries of these 3 cases, let us recall that $\mu=1$ corresponds, via (17), to $g=0$, namely, see (3), to the trivially solvable two-body case, see (5), while $\mu=0$ respectively $\mu=\infty$ correspond, via (17), to $f+2 g=0$ respectively to $f+8 g=0$ and require a separate treatment, for which the interested reader is referred to [4]. Clearly the third case $(\mu<0)$ becomes identical to the first $(\mu>1)$ via the replacement

$$
\tilde{w} \mapsto 1-\tilde{w}, \quad \bar{\xi} \mapsto-\bar{\xi}, \quad R \mapsto-R, \quad-p \mapsto p-q, \quad q-p \mapsto p
$$

without modifying $q$; therefore in the following, without loss of generality, we often forsake a separate discussion of this third case.

Clearly the factor $[\bar{\xi}+R \exp (2 \mathrm{i} \omega t)]^{q}$, which carries all the time dependence in these polynomial equations, is periodic in $t$ with period $T$, see (2) (except for the special initial conditions entailing $\bar{\xi}=0$, in which case this factor is instead periodic with the shorter period $T / q$ ) (for simplicity we continue to pursue our policy to consider only the generic case when this does not happen, referring the interested reader to [4] for a more complete treatment). At issue is the behaviour of the $J$ roots $\tilde{w}_{j}(t)$ of our polynomial equation $(21)$ whose coefficients evolve in time periodically with period $T$. Let us indicate with $\tilde{W}(t) \equiv\left\{\tilde{w}_{j}(t) ; j=1, \ldots, J\right\}$ the (unordered) set of these $J$ roots. Obviously $\tilde{W}(t)$ is periodic with period $T, \tilde{W}(t+T)=\tilde{W}(t)$ : after one period $T$ the polynomial equation is unchanged, hence the set of its $J$ roots is as well unchanged. But that does not imply that if one follows the time evolution of these $J$ roots, each of them will return to its own initial value after one period, $\tilde{w}_{j}(T)=\tilde{w}_{j}(0), j=1, \ldots, J$. This outcome will indeed obtain for the open domain of initial data of our problem that corresponds to the basic isochronous regime, see (10); but it does not happen for other initial data, in which cases for instance a generic root, say $\tilde{w}_{j_{1}}(t) \equiv \tilde{w}(t)$, may after one period land in the initial position of a different root, say $\tilde{w}(T)=\tilde{w}_{j_{2}}(0)$, and then after one more period end up in the initial position of yet another root, $\tilde{w}(2 T)=\tilde{w}_{j_{3}}(0)$, and so on. Eventually, of course, after a time $\tilde{T}=\tilde{j} T$ which is a finite integer multiple $\tilde{j}$ of the basic period $T$, with $1 \leq \tilde{j} \leq J$, the generic root $\tilde{w}(t)$ shall necessarily return to its initial position, $\tilde{w}(\tilde{T})=\tilde{w}(0)$, entailing that its evolution as a function of the time $t$ is periodic with this period $\tilde{T}$,

$$
\tilde{w}(t+\tilde{T}) \equiv \tilde{w}(t+\tilde{j} T)=\tilde{w}(t)
$$

This discussion clearly implies (via (13a) ) that, in the case now under consideration (with a rational value of the parameter $\mu$, see (20)), all solutions of our physical problem (1) with (3) are completely periodic with a period which is either

$$
\check{T}=\check{j} T \quad \text { with } 1 \leq \check{j} \leq J
$$


and $J$ defined by (22), or it is $2 \check{T}$ (see the discussion above following (19)). The remaining, crucial question is: how does the value of the integer $\check{j}$ (which might be quite large if $J$ is quite large) depend on the initial data of our problem? In this paper we outline how to calculate, for given initial data, all the possible periods of the $J$ roots $\tilde{w}_{j}(t)$ of $(21)$, and we display formulas providing (at most) 3 alternative values for these periods; as already mentioned above, the explanation of how to identify which one of these 3 periods corresponds to that of the "physical" root $\check{w}(t)$ entails a more detailed treatment which is reported in [4].

But before doing so let us emphasize that via this discussion the time evolution of our original 3-body problem - describing the time evolution of the three points $z_{n}(t)$ in the complex $z$-plane - has been related to the time evolution of the $J$ roots $\tilde{w}_{j}(t)$ of (21) in the complex $w$-plane, and in particular to the way they get permuted among themselves over the time evolution after each period $T$. As we will explain below, the possible complications of the motions of our physical model (1) are thereby related to the mechanisms at play to permute these roots among themselves when one watches their time evolution at subsequent intervals $T, 2 T, 3 T$ and so on. And, in this context, it is significant to note that, whenever $\mu$ is irrational, one is in fact dealing with the dynamics of an infinite number of roots. This suggests that whenever the number $J$ of roots is large, and even more so when $\mu$ is irrational (entailing $J=\infty$ ), the time evolution of our physical model (11) might be quite complicated, perhaps calling into play the theoretical tools of statistical mechanics rather than those of few-body dynamics (but we postpone such excursions to future publications).

As entailed by our discussion above, the issue of determining the value of the integer $\tilde{j}$ is tantamount to understanding the structure of the Riemann surface associated with the function $w(\xi)$ of the complex variable $\xi$ defined by the nondifferential equation (16), whose different sheets correspond of course to the different roots of this nondifferential equation. In the rational case (20) this equation is in fact polynomial of degree $J$ in the dependent variable $w$. Specifically, what must be ascertained is the number of sheets of this Riemann surface that are accessed by $w(\xi)$ when the independent variable $\xi$ travels in the complex $\xi$-plane round and round on the circle $\Xi$, whose center $\bar{\xi}$ and radius $|R|$ depend on the initial data of our physical problem, see (15) and (18). To this end one must gain and use a detailed understanding of the structure of this Riemann surface. Again, we refer for the details of this analysis to [4]. Suffice here to report the following basic information on the analytic structure of the function $w(\xi)$, referring to the general case with real $\mu$ (rational or irrational).

The nondifferential equation (16) defines a $J$-sheeted covering of the complex $\xi$ plane of genus zero (of course $J=\infty$ is $\mu$ is irrational). The function $w(\xi)$ defined implicitly by this equation features square-root branch points $\xi_{b}$ located on a circle $B$ centered at the origin of the complex $\xi$-plane:

$$
\begin{aligned}
& \xi_{b}=\xi_{b}^{(k)}=r_{b} \exp (2 \pi \mathrm{i} \mu k), \quad k=1,2,3, \ldots, \\
& \xi_{b}=\xi_{b}^{(k)}=r_{b} \exp \left[\mathrm{i} \frac{2 \pi p k}{q}\right], \quad k=1,2, \ldots, q, \\
& r_{b}=(\mu-1)^{-1}\left(\frac{\mu-1}{\mu}\right)^{\mu} .
\end{aligned}
$$

In the last, (26c), of these formulas it is understood for definiteness that the principal determination is taken of the $\mu$-th power appearing in the right-hand side. The first of these formulas, (26a), shows clearly that the number of these branch points is infinite 
if the parameter $\mu$ is irrational, and that they then sit densely on the circle $B$ in the complex $\xi$-plane centered at the origin and having radius $\left|r_{b}\right|$, see (26c). Note that this entails that the generic point on the circle $B$ is not a branch point (just as a generic real number is not rational); but every generic point on the circle $B$ has, if $\mu$ is irrational, some branch point (in fact, an infinity of branch points!) arbitrarily close to it (just as every generic real number has an infinity of rational numbers arbitrarily close to it). It is also important to realize that these branch points are generally on different sheets of the Riemann surface associated with the function $w(\xi)$ : hence, they are dense if one considers the circle $B$ in the complex $\xi$-plane, but they are not dense if one considers these branch points on the Riemann surface itself. As for the second of these formulas, (26b), it is instead appropriate to the case in which the parameter $\mu$ is rational, see (20), in which case the branch points sit again on the circle $B$ in the complex $\xi$-plane, but there are only a finite number, $q$, of them (and note that the factor $p$ appearing in the argument of the exponential in the right-hand side of this formula, (26b), is only relevant to characterize how the branch points $\xi_{b}^{(k)}$ are labeled via the index $k$ ). Both in the irrational and in the rational case at all these branch points the nondifferential equation (16) has a double root which takes the same value $w\left(\xi_{b}\right)=\mu$. Note that this entails that circling around such a branch point in the complex $\xi$-plane corresponds to permuting 2 of the $J$ roots $w_{j}(\xi)$ among themselves.

In addition, the function $w(\xi)$ possesses branch points at $\xi=0$ and at $\xi=\infty$, the order of which depends on the value of $\mu$, and is rational if the number $\mu$ is rational (see below).

The branch point at $\xi=\infty$ has, if $\mu>1$, exponent $-\frac{1}{\mu}\left(=-\frac{q}{p}\right.$ in the rational case),

$$
\xi \approx \infty, \quad w(\xi) \approx a \xi^{-1 / \mu} \approx 0, \quad a^{\mu}=-\exp (-\mathrm{i} \pi \mu), \quad \mu>0,
$$

while it has instead two different exponents if $0<\mu<1$ :

(i) the exponent $-\frac{1}{\mu}\left(=-\frac{q}{p}\right.$ in the rational case) as given by the preceding formula (entailing $w \approx 0$ )

(ii) the exponent $-\frac{1}{1-\mu}\left(=\frac{q}{q-p}\right.$ in the rational case) as given by the following formula (entailing $w \approx 1$ ),

$$
\xi \approx \infty, \quad w(\xi) \approx 1+a \xi^{-1 /(1-\mu)} \approx 1, \quad a^{\mu-1}=1, \quad 0<\mu<1 .
$$

The branch point at $\xi=0$ is, if $\mu>1$, of exponent $\frac{1}{\mu-1}\left(=\frac{q}{p-q}\right.$ in the rational case),

$$
\xi \approx 0, \quad w(\xi) \approx 1+a \xi^{1 /(\mu-1)} \approx 1, \quad a^{\mu-1}=1, \quad \mu>1,
$$

(note the formal analogy of this formula with the previous one, (28)), and it is instead absent if $0<\mu<1$, so that in this second case the only branch points in the finite part of the complex $\xi$-plane are those of square-root type, see (26a). This is the main cause of the difference between the results, see below, for this case $(0<\mu<1)$ from those for the other two cases $(\mu>1$ and $\mu<0)$, which are on the other hand essentially equivalent among each other being related by the transformation $\mu \mapsto 1-\mu$, $w(\xi) \mapsto 1-w(-\xi)$, see (16) (so that without loss of generality we often forsake an explicit discussion of the case with $\mu<0$ ).

Note that these results entail that, in the rational case, see (20), making a circle around the branch point at $\xi=0$, in the $p>q$ (i. e. $\mu>1$ ) case when this branch point is present, causes a cyclic permutation of $p-q$ of the $p$ roots $w_{j}$ : this 
is particularly evident if one imagines to travel full circle around the branch point at $\xi=0$ in its immediate vicinity, since for $\xi \approx 0$ the $p$ roots $w_{j}$ of (16) are clearly divided into two sets, a first set of $p-q$ roots, disposed equispaced on a circle of small radius $\left(\approx|\xi|^{q /(p-q)}\right)$ centered at $w=1$ in the complex $w$-plane, which then undergo a cyclic permutation among themselves, and a second set of $q$ roots, disposed equispaced on a circle in the complex $w$-plane centered at the origin and having a large radius $\left(\approx|\xi|^{-1}\right)$, each of which after the operation returns instead to its original position.

The permutation experienced by the $J$ roots $\tilde{w}_{j}(t)$ due to a sequence of pairwise exchanges of roots - which take place whenever square-root branch points are included inside the circle $\Xi$ traveled by the point $\xi(t)$ - causes a reshuffling of the roots which is nontrivial inasmuch as it depends on how many and on which pairs of roots get sequentially exchanged over each period, as determined by the number and identity of square-root branch points enclosed inside the circle $\Xi$ traveled by $\xi(t)$ and by the detailed structure of the Riemann surface associated with these branch points, in particular on which sheets of this Riemann surface the relevant branch points are located. The reshuffling encompasses more roots when a second mechanism is simultaneously at play, i. e. that producing a cyclic permutation of $p-q$ roots (especially, of course, when $p-q$ is large) over each period, as caused by the presence of the branch point at $\xi=0$ : this second mechanism exists only if $\mu>1$ (or $\mu<0$, entailing the exchange $-p \mapsto p-q$ ), and provided the circle $\Xi$ traveled by $\xi(t)$ does include the point $\xi=0$. This phenomenology causes the possible periods $\tilde{T}=\tilde{j} T$ of the time evolution of the generic root $\tilde{w}(t)$ to depend on the initial data, but remarkably we will see that, for given initial data, there are (at most) only 3 possible values of these periods, and that they can be given explicitly in terms of the initial data and of the two numbers $p$ and $q$, see (20). Indeed we show below that analogous results can as well be given in the case when $\mu$ is irrational, in spite of the fact that the dependence on the initial data may then be quite sensitive.

The correspondence of the analysis, given here in terms of the dynamics of the roots $\tilde{w}_{j}(t)$, with that in terms of travel on the Riemann surface made above (see paragraphs after (12)), including the distinction made there about active and inactive branch points, should be noted: the active branch points are those that cause a reshuffling of roots that involves the "physical" root $\breve{w}(t)$, the inactive ones are those that do not cause a reshuffling of roots that involves the "physical" root $\check{w}(t)$, either because they cause no reshuffling at all being located outside the relevant circle $(\Xi$ in the complex $\xi$-plane in the context of the present analysis, $C$ in the complex $\tau$-plane in the context of the discussion made above when the distinction among active and inactive branch points was first introduced), or because they cause a reshuffling which however does not involve the physical root $\check{w}(t)$.

We now report our findings concerning the time evolution of a generic root $\tilde{w}(t)$, referring at first mainly to the rational case but including immediately results for the irrational case whenever it is convenient to do so in order to shorten our presentation. The remaining information on the irrational case is provided below (see Proposition $5)$.

First of all it is useful to visualize the two circles $B$ and $\Xi$ in the complex $\xi$-plane (draw them!): recall that the circle $B$ on which the branch points sit is centered at the origin and its radius $\left|r_{b}\right|$ only depends on the parameter $\mu$, see (26c), while both the center $\bar{\xi}$ and the radius $|R|$ of the circle $\Xi$ traveled upon by $\xi(t)$, see (15), do depend on the initial data, see (18). 
Proposition 1. If the circle $\Xi$ is inside the circle $B$ (i. e. $|\bar{\xi}|+|R|<\left|r_{b}\right|$, see (18) and (26c) $)$, and (a) $\mu$ is inside the interval $0<\mu<1$ or (b) $\mu$ is outside this interval $(\mu>1$ or $\mu<0)$ and moreover the circle $\Xi$ does not include the origin $\xi=0$ (i. e. $|R|<|\bar{\xi}|$ or equivalently $|\eta|>1$ ), then $\tilde{j}=1$, i. e. the generic solution $\tilde{w}(t)$ is periodic with period $T, \tilde{w}(t+T)=\tilde{w}(t)$. This outcome applies equally if $\mu$ is rational or irrational.

Remark 1. In all the cases identified in this Proposition 1 there are no branch points at all inside the circle $\Xi$ : indeed the outcome detailed by this Proposition 1 applies in all the cases in which this happens (see our discussion above), even (in the case with rational $\mu$ ) if the circles $B$ and $\Xi$ do cross each other marginally; and of course in all these cases all the roots $\tilde{w}_{j}(t)$ are periodic with period $T$, and in the context of the physical problem (11) the solution is characterized by the simple periodicity rule (10). The restriction (12) on the initial data is sufficient (but of course not necessary) to guarantee that we are in this regime. $\square$

Proposition 2. If the circle $\Xi$ is inside the circle $B$ (i. e. $|\bar{\xi}|+|R|<\left|r_{b}\right|$ ), the circle $\Xi$ does include the origin $\xi=0$ (i. e. $|R|>|\bar{\xi}|$ or equivalently $|\eta|<1$ ), and $\mu$ is outside the interval $0<\mu<1$ then in the rational case, see (20),

(a) $\tilde{j}=1$ or $\tilde{j}=p-q$ if $\mu>1$

(b) $\tilde{j}=1$ or $\tilde{j}=|p|$ if $\mu<0$.

In the irrational case the time evolution of the generic root $\tilde{w}(t)$ is either periodic with the basic period $T$, or quasiperiodic, involving in particular a (nonlinear) superposition of two periodic evolutions with two noncongruent periods, specifically (a) with period $T$ and $\frac{T}{\mu-1}$ if $\mu>1$, (b) with period $T$ and $\frac{T}{|\mu|}$ if $\mu<0$.

Remark 2. In the case identified in this Proposition 2 the only branch point inside $\Xi$ is that at $\xi=0$, which is indeed only present if $\mu>1$ or $\mu<0$. Hence in the rational case with $p>q$ (i. e. $\mu>1), p-q$ roots $\tilde{w}_{j}(t)$ get cyclically exchanged among themselves, entailing that the time evolution of each of them has period $(p-q) T$, while the remaining $q$ roots have period $T$; with an analogous phenomenology in the $\mu<0$. Likewise, when $\mu$ is irrational, the periodicity of the time evolution of the generic root $\tilde{w}(t)$ has period $T$ if the branch point at $\xi=0$ is inactive (i. e., it does not appear on the sheet on which $\tilde{w}(0)$ lives), otherwise its time evolution can be inferred by replacing $\xi$ with $\xi(t)$, see (15), in the formula characterizing the branch point at $\xi=0$, see (29). Note however that for the special initial data such that the two circles $B$ and $\Xi$ are concentric (i. e., $\eta=0$ ) the quasiperiodic time evolution of $\tilde{w}(t)$ is instead periodic (a) with period $\frac{T}{\mu-1}$ if $\mu>1$, (b) with period $\frac{T}{|\mu|}$ if $\mu<0$. $\square$

Proposition 3. If the circle $\Xi$ is outside the circle $B$ (i. e. $|R|>|\bar{\xi}|+\left|r_{b}\right|$ ) then in the rational case, see (20),

(a) $\tilde{j}=p$ if $\mu>1$,

(b) $\tilde{j}=q-p=q+|p|$ if $\mu<0$

(c) $\tilde{j}=p$ or $\tilde{j}=q-p$ if $0<\mu<1$.

In the irrational case the time evolution of the generic root $\tilde{w}(t)$ is quasiperiodic, involving a (nonlinear) superposition of two periodic evolutions with two noncongruent periods, specifically

(a) with periods $T$ and $\frac{T}{\mu}$ if $\mu>1$,

(b) with periods $T$ and $\frac{T}{1-\mu}$ if $\mu<0$, 
(c) with periods $T$ and $\frac{T}{\mu}$ or $T$ and $\frac{T}{1-\mu}$ if $0<\mu<1$.

Remark 3. In all the cases encompassed in this Proposition 3 the branch points of $w(\xi)$ in the finite part of the complex $\xi$-plane are all inside the circle $\Xi$, hence the dynamics of the roots $\tilde{w}_{j}(t)$ can be understood in terms of the branch point of $w(\xi)$ at $\xi=\infty$. Therefore in the rational case with $p>q$ (i. e. $\mu>1$ ) all the $p$ roots get cyclically exchanged, so that each of them gets back to its original value after a period $p T$; likewise if $p<0$ (i. e. $\mu<0$ ) all the $q+|p|$ roots get cyclically exchanged, so that each of them gets back to its original value after a period $(q+|p|) T$. In the other rational case, $0<p<q$ (i. e. $0<\mu<1$ ), $p$ roots gets cyclically exchanged among themselves, and the remaining $q-p$ roots get cyclically exchanged among themselves, so that the generic root $\tilde{w}(t)$ has period $p T$ if it belongs to the first set, and $(q-p) T$ if it belongs to the second. And the outcome in the irrational case can as well be understood in terms of the exponent of the branch point at $\xi=\infty$, see (27)-(28).

The situation is less straightforward (hence more interesting) if the two circles $B$ and $\Xi$ do intersect each other (i. e. $|\bar{\xi}|-|R|<\left|r_{b}\right|<|\bar{\xi}|+|R|$ ). Then the parameter that plays a crucial role is the number $b$ of square-root branch points, sitting on the circle $B$, that fall inside the circle $\Xi$. This number $b$ is finite, $1 \leq b \leq q$ (note that the case $b=0$ is taken care of by Proposition 1) only in the rational case, to which we restrict consideration in the following Proposition 4 (and we exclude from consideration the nongeneric case in which the circle $\Xi$ hits one of the branch points sitting on the circle $B$ ). Then two or three (but no more!) different alternatives are possible for the value of the positive integer $\tilde{j}$, as detailed below.

Hereafter the notation $\lfloor x\rfloor$ denotes the floor of the real number $x$, namely the largest integer number not larger than $x$ (hence for instance $\lfloor-0.3\rfloor=-1,\lfloor 0\rfloor=0$ ).

Proposition 4. (i). If $p>q$ (i. e. $\mu>1$, see (201) ) and the origin $\xi=0$ is outside the circle $\Xi$ (i. e. $|\eta|>1$ ), then $\tilde{j}$ can take one of the following 3 values:

$$
\tilde{j}=1 \quad \text { or } \quad \tilde{j}=\left\lfloor\frac{b-1}{p-q}\right\rfloor+1 \quad \text { or } \quad \tilde{j}=\left\lfloor\frac{b-1}{p-q}\right\rfloor+2 .
$$

(ii). If $p>q_{\sim}$ (i. e. $\mu>1$, see (20) ) and the origin $\xi=0$ is inside the circle $\Xi$ (i. e. $|\eta|<1)$, then $\tilde{j}$ can take one of the following 2 values:

$$
\tilde{j}=1 \text { or } \tilde{j}=b+p-q \text {. }
$$

(i'). If $p<0$ (i. e. $\mu<0$, see (201) ) and the origin $\xi=0$ is outside the circle $\Xi$ (i. e. $|\eta|>1$ ), then $\tilde{j}$ can take one of the following 3 values:

$$
\tilde{j}=1 \quad \text { or } \quad \tilde{j}=\left\lfloor\frac{b-1}{|p|}\right\rfloor+1 \quad \text { or } \quad \tilde{j}=\left\lfloor\frac{b-1}{|p|}\right\rfloor+2 .
$$

(ii'). If $p<0$ (i. e. $\mu<0$, see (201) ) and the origin $\xi=0$ is inside the circle $\Xi$ (i. e. $|\eta|<1$ ), then $\tilde{j}$ can take one of the following 2 values:

$$
\tilde{j}=1 \text { or } \tilde{j}=b+|p| .
$$

(iii). The situation is more intriguing if $0<p<q$ (i. e., $0<\mu<1$, see (20). Then one must introduce the simple continued fraction expansion of the number

$$
\frac{q}{q-p}=\frac{1}{1-\mu}=a_{0}+\frac{1}{a_{1}+\frac{1}{a_{2}+\ldots}} .
$$


The $k$ th convergent $C_{k}$ of this continued fraction expansion (34), and in particular its numerator $P_{k}$ and denominator $Q_{k}$,

$$
C_{k}=\frac{P_{k}}{Q_{k}}
$$

are then defined by the recursions

$$
\begin{array}{llll}
P_{k}=a_{k} P_{k-1}+P_{k-2}, & P_{-2}=0, & P_{-1}=1, & k=0,1,2, \ldots \\
Q_{k}=a_{k} Q_{k-1}+Q_{k-2}, & Q_{-2}=1, & Q_{-1}=0, & k=0,1,2, \ldots
\end{array}
$$

Note that these formulas apply equally if $\mu$ is rational or irrational; of course depending whether $\mu$ is rational or irrational the continued fraction expansion does or does not terminate. In the rational case under present discussion we also introduce another sequence of nonnegative integers:

$$
b_{k}=q-(-)^{k}\left[(p-q) P_{k-2}+q Q_{k-2}\right], \quad k=0,1,2, \ldots
$$

Given $b$, let the integer $h$ and the period $T(b)$ be defined by the following formulas:

$$
\begin{aligned}
& b_{h} \leq b<b_{h+1}, \\
& T(b)=P_{h-2}+\left(\left\lfloor\frac{b-b_{h}-1}{q-b_{h+1}}\right\rfloor+2\right) P_{h-1} .
\end{aligned}
$$

Then the roots of the polynomial can have only one of the following three periods $\tilde{j}$ :

$$
\tilde{j}=T(b) \quad \text { or } \quad \tilde{j}=T(b)-P_{h-1} \quad \text { or } \quad \tilde{j}=P_{h-1} .
$$

This is the generic case; there are however some cases in which the roots have only the two periods $T(b)$ and $P_{h-1}$. This happens of course when $T(b)=2 P_{h-1}$ and whenever $b$ takes the following special values:

$$
b=b_{h}+n\left(q-b_{h+1}\right), \quad 0 \leq n \leq a_{h}-1, \quad n \text { integer. }
$$

Remark 4. In case (i) of Proposition 4 the mechanism that yields periods longer than unity is the coming into play of the $b$ square-root branch points enclosed inside the circle $\Xi$, which cause a certain number of roots $\tilde{w}_{j}(t)$ to exchange pairwise their roles through the time evolution. But this phenomenology only affects some roots; others remain unaffected, hence their periods remain unity, and this explains the first entry in (30). The precise form of the other entries in this formula, (30), requires of course a more detailed treatment, see [4]; the outcome there depends on how many pair exchanges actually do take place, or, equivalently, how many sheets of the Riemann surface get actually visited, and this depends in a fairly detailed manner on the specific structure of this surface. But note that only two different periods may emerge, differing by only one unit.

In case (ii) of Proposition 4 the second mechanism, associated with the presence of the branch point at $\xi=0$, comes additionally into play, causing the connection of all the $b$ sheets containing the $b$ square-root branch points, both among themselves and with the $(p-q)$ sheets containing the branch point at $\xi=0$. The corresponding $(b+p-q)$ roots get permuted among themselves through the time evolution, with the period indicated by the second entry in (31) (note that whenever $p$ is quite large and $b$ is close to its maximal value $q-1$, the resulting period is quite large). The remaining $(q-b)$ sheets, corresponding to the $(q-b)$ branch points lying on the circle $B$ outside the circle $\Xi$, are isolated, hence the corresponding roots do not take part in the quadrille, so that their period remains unity, as indicated by the first entry in (31). 
The cases (i') and (ii') of Proposition 4 require no additional discussion.

In case (iii) of Proposition 4 (with $0<p<q$, i. e. $0<\mu<1$ ) there is no branch point at $\xi=0$; hence the mechanism is now absent that previously caused the connection of all the sheets associated with the $b$ square-root branch points sitting on the circle $B$ inside the circle $\Xi$. This implies, see [4], that each sheet of the Riemann surface contains only two branch points, whose projections on the circle $B$ are separated by $(p-q)$ other branch points. Note that this entails that two branch points which are adjacent on the circle $B$ in the complex $\xi$-plane are instead topologically far apart on the Riemann surface of the function $w(\xi)$, living on sheets which are not directly connected. As a consequence the period of the time evolution of the root $\tilde{w}(t)$ does not change, as the initial data change causing the circle $\Xi$ to change its position and dimension so that the number $b$ of square-root branch points enclosed in it increases, until some crucial square-root branch point gets thereby included inside the circle $\Xi$, causing the connection of two separate groups of connected sheets; and this mechanism occurs more and more frequently as $b$ increases more and more. This explained qualitatively the piecewise constant behavior of the period as $b$ increases, characterized by shorter and shorter steps and by bigger and bigger jumps, see (40)(41). The exact treatment of this mechanism, yielding (40)-(41), is rather complicated, as indicated by the role played by the continued fraction expansion of the number $\frac{q}{q-p}$. The details are given in [4]. Here we limit ourselves to emphasizing that, for given initial data, the generic root $\tilde{w}(t)$ can have only 3 possible periods, the third of which is just the sum of the first two, see (41).

Let us now discuss the case in which $\mu$ is an irrational number, recalling that we are now considering initial data $\left(|\bar{\xi}|-|R|<\left|r_{b}\right|<|\bar{\xi}|+|R|\right)$ such that the two circles $B$ and $\Xi$ do intersect each other (the results for the other cases have been given in Propositions 1-3. ). One must then introduce the ratio $\nu$ of the length of the arc of the circle $B$ that is inside the circle $\Xi$, to the length of the entire circle $B$ : of course $0<\nu<1$. Expressing this ratio in terms of the initial data is an exercise in elementary plane geometry yielding the formula

$$
\nu=\frac{1}{\pi} \arccos \left(\frac{\left|r_{b}\right|^{2}+|\bar{\xi}|^{2}-|R|^{2}}{2\left|r_{b} \bar{\xi}\right|}\right),
$$

where the determination of the arccos function must be chosen so that $0<\nu<1$. The results for the periods are then given by the following

Proposition 5. (i) If $\mu>1$ and the origin $\xi=0$ is outside the circle $\Xi$ (i. e. $|\eta|>1)$, the time evolution of the generic $\operatorname{root} \tilde{w}(t)$ is still periodic with period $\tilde{T}=$ $\tilde{j} T$ and $\tilde{j}$ can take one of the following 3 values:

$$
\tilde{j}=1 \quad \text { or } \quad \tilde{j}=\left\lfloor\frac{\nu}{\mu-1}\right\rfloor+1 \text { or } \tilde{j}=\left\lfloor\frac{\nu}{\mu-1}\right\rfloor+2 .
$$

Note that the second and third entry only differ by one unit and moreover that, if $\mu>2$, the floor functions vanish, hence for all values of $\mu$ larger than 2 (and $|\eta|>1$ ) the only possible values for $\tilde{j}$ are 1 or 2 .

(ii) If $\mu>1$ and the origin $\xi=0$ is inside the circle $\Xi$ (i. e. $|\eta|<1$ ), the time evolution of the generic root $\tilde{w}(t)$ is either periodic with period $T$ or aperiodic.

(i'). If $\mu<0$ and the origin $\xi=0$ is outside the circle $\Xi$ (i. e. $|\eta|>1$ ), the time evolution of the generic root $\tilde{w}(t)$ is still periodic with period $\tilde{T}=\tilde{j} T$ and $\tilde{j}$ can take one of the following 3 values:

$$
\tilde{j}=1 \quad \text { or } \quad \tilde{j}=\left\lfloor\frac{\nu}{|\mu|}\right\rfloor+1 \quad \text { or } \quad \tilde{j}=\left\lfloor\frac{\nu}{|\mu|}\right\rfloor+2 .
$$


Again the second and third entry only differ by one unit and moreover if $|\mu|>2$ the floor functions vanish, hence we conclude that for all values of $\mu$ smaller than -2 (and $|\eta|>1$ ) the only possible values for $\tilde{j}$ are 1 or 2 .

(ii') If $\mu<0$ and the origin $\xi=0$ is inside the circle $\Xi$ (i. e. $|\eta|<1$ ), the time evolution of the generic root $\tilde{w}(t)$ is either periodic with period $T$ or aperiodic.

(iii). The case $0<\mu<1$ is again more intriguing, and it requires again the use of the continued fraction expansion of $\frac{1}{1-\mu}$, which however now does not terminate. We define now in addition the (endless) sequence of real numbers

$$
\nu_{k}=1-(-)^{k}\left[(\mu-1) P_{k-2}+Q_{k-2}\right], \quad k=0,1,2, \ldots
$$

(entailing $\nu_{0}=0$ ), and we then identify the nonnegative integer $h$ via the inequalities

$$
0 \leq \nu_{h} \leq \nu<\nu_{h+1}<1 \text {. }
$$

Let $T(\nu)$ be defined by the following expression:

$$
T(\nu)=P_{h-2}+\left(\left\lfloor\frac{\nu-\nu_{h}}{1-\nu_{h+1}}\right\rfloor+2\right) P_{h-1} .
$$

Then the motion of the generic root $\tilde{w}(t)$ is again periodic with period $\tilde{T}=\tilde{j} T$ where $\tilde{j}$ can take one of the following 3 values:

$$
\tilde{j}=T(\nu) \quad \text { or } \quad \tilde{j}=T(\nu)-P_{h-1} \quad \text { or } \quad \tilde{j}=P_{h-1} .
$$

Remark 5. The results for this case with irrational $\mu$ can be obtained from those for rational $\mu$ (see Proposition 4) by taking appropriately the limit in which

(a) the integers $p$ and $q$ diverge with their ratio $\mu$ fixed (see (201) ), and

(b) the number $b$ of square-root branch points inside the circle $\Xi$, as well as the total number $q$ of square-root branch points, also diverge with their ratio fixed (recall that all these square-root branch points sit, densely equispaced, on the circle $B$ in the complex $\xi$-plane although on different sheets of the Riemann surface of the function $w(\xi)$ - hence this ratio coincides with the quantity $\nu$ defined, and evaluated in terms of the initial data, above, see (43)).

This also suggests obvious extensions to the present case with irrational $\mu$ of comments contained in the Remark 4, which will not be repeated here. We therefore limit below our remarks to aspects of the results reported in Proposition 5 having no immediate counterpart in the comments contained in Remark 4.

In the cases (i) respectively (i') of Proposition 5 the rules giving the period of the time evolution of the generic root $\tilde{w}(t)$, see (44) respectively (45), are fairly straightforward and generally yield rather small periods, unless $\mu=1+\varepsilon$ respectively $\mu=-\varepsilon$ with $\varepsilon$ an irrational number positive but extremely small.

In cases (ii) and (ii') of Proposition 5 the situation is quite interesting because the time evolution of the generic root $\tilde{w}(t)$ can be either periodic with the basic period $T$ or aperiodic. Note that in these cases the circle $\Xi$ intersects the circle $B$ that is densely filled with square-root branch points, and moreover the branch point at $\xi=0$ (which is now of irrational exponent, see (29)) is inside the circle $\Xi$. This entails that, of the infinity of square-root branch points located on the piece of the circle $B$ that is inside $\Xi$, either none, or all, are active. The first case obtains if the root $\tilde{w}(t)$ under consideration is initially on a sheet containing a branch point that does not fall inside $\Xi$, hence the time evolution of this root $\tilde{w}(t) \equiv w[\xi(t)]$ as the point $\xi(t)$ travels round and round on the circle $\Xi$ brings it back to its point of departure after 
a single round; equivalently, in this case the root $\tilde{w}(t) \equiv w[\xi(t)]$ belongs to a set of roots that does not get permuted as the point $\xi(t)$ travels round and round on the circle $\Xi$. In the second case the root $\tilde{w}(t)$ under consideration starts from a sheet of the Riemann surface that contains a branch point inside $B$, so that, when $\xi(t)$ travels round and round on the circle $\Xi$, an endless sequence of different sheets get accessed by $\tilde{w}(t) \equiv w[\xi(t)]$; equivalently, such a root $\tilde{w}(t) \equiv w[\xi(t)]$ belongs to a set (including an infinity of roots) that does get permuted as the point $\xi(t)$ travels round and round on the circle $\Xi$, with both mechanisms - the pairwise exchange of some roots, and the cyclic permutation of an infinite number of roots - playing a role at each round. The identification of which sheets get thereby accessed, and in which order - namely the specific shape of the trajectory when looked at, as it were stroboscopically, at the discrete sequence of instants $T_{k}=k T, k=1,2,3, \ldots-$ is discussed in [4]. The extent to which this regime yields irregular motions is discussed further below, also to illuminate the distinction in these regimes between the time evolution entailed by our model with a given irrational value of $\mu$, and that of the analogous models with rational values of $\mu$ providing more and more accurate approximations of the given irrational value of $\mu$.

In case (iii) the time evolution is still isochronous, inasmuch as the results reported above entail that, for any given initial data (excluding, of course, the special ones leading to a collision; which are special in the same sense as a rational number is special in the context of real numbers), the motion of every root $\tilde{w}_{j}(t)$ is periodic with one of the 3 periods entailed by (49) (and note that the value of the integer $\tilde{j}$ provided by the third of these 3 formulas is just the sum of the 2 values for $\tilde{j}$ provided by the first 2 of these 3 formulas). It is indeed clear that the initial data yielding such an outcome are included in an open set of such data, having of course full dimensionality in the space of initial data, all yielding the same outcome: since the periods do not change, see (49), if the change of the initial data, hence the change in the ratio $\nu$, is sufficiently tiny. However the measures of these sets of data yielding the same outcome gets progressively smaller as the predicted periods get larger, and moreover the corresponding predictions involve more and more terms in the (never ending) continued fraction expansion of the irrational number $\frac{1}{1-\mu}$, see (34), displaying thereby, as $\nu$ increases towards unity, a progressively more sensitive dependence of the periodicity of our system on the initial data and moreover on the parameters (the coupling constants, that determine the value of $\mu$, see (17)) of our physical model (11). $\square$

Example. Let us display here a specific example with the following (conveniently chosen) irrational value of $\mu$ in the interval $0<\mu<1$ (hence corresponding to case (iii) of Proposition 5):

$$
\mu=\frac{2}{3+\sqrt{5}}=\frac{1}{1+\varphi}, \quad \frac{1}{1-\mu}=\varphi=\frac{1+\sqrt{5}}{2},
$$

where of course $\varphi$ is the golden ratio. This assignment entails that all the coefficients $a_{k}$, see (34), are in this case unity, $a_{k}=1$, hence the quantities $P_{k}$, see (37), coincide with the Fibonacci numbers $F_{k}$,

$$
\begin{aligned}
& P_{k}=F_{k}, \quad F_{-2}=0, \quad F_{-1}=1, \quad F_{0}=1, \\
& F_{k+2}=F_{k}+F_{k+1},
\end{aligned}
$$

and moreover one easily finds that

$$
\nu_{k}=1-\varphi^{-k} \text {. }
$$


The corresponding formula for the periods obtains inserting these values in (47) and (49). From it, with some labor, one can obtain the following controlled estimate for the possible values of $\tilde{j}$ :

$$
\frac{\nu(2-\nu)}{\sqrt{5}(1-\nu)} \leq \tilde{j} \leq \frac{\sqrt{5}+2-(\sqrt{5}+1) \nu(2-\nu)}{(\sqrt{5}-1)(1-\nu)}
$$

These inequalities are valid for all values of $\nu$ (in the interval $0<\nu<1$ ); they clearly entail that the integer $\tilde{j}$ diverges proportionally to $(1-\nu)^{-1}$ as $\nu \rightarrow 1$, and that for $0<\nu<\bar{\nu}$ with

$$
\bar{\nu}=\sqrt{5}-2+\frac{3-\sqrt{5}}{2} \sqrt{3-\frac{\sqrt{5}}{2}}=0.760067 \ldots
$$

the only possible values of $\tilde{j}$ are 2 and 3 .

This concludes our presentation of the results, and of some related observations, detailing the periodicity (if any) of the time evolution of a generic root $\tilde{w}(t)$ of (16) with (15). The identification of analogous, but of course more definite, results for the physical root $\check{w}(t)$, and the consequential information on the periodicity (if any) of the solution of the physical problem (1) - as well as some additional information on the corresponding trajectories of the coordinates $z_{n}(t)$ - are provided in [4].

Last but not least let us elaborate on the character of the aperiodic time evolution indicated under item (ii) of Proposition 5, including the extent it is irregular and it depends sensitively on its initial data. It is illuminating to relate this question with the finding reported under item (ii) of Proposition 4, also in order to provide a better understanding of the relationship among the aperiodic time evolution that can emerge when $\mu$ is irrational (see item (ii) of Proposition 5) and the corresponding behavior - say, with the same initial data - for a sequence of models with rational values of $\mu$ (see [20) that provide better and better approximations to that irrational value of $\mu$; keeping in mind the qualitative difference among the aperiodic time evolution emerging when $\mu$ is irrational, and the periodic - indeed, even isochronous - time evolutions prevailing whenever $\mu$ is rational, albeit with the qualifications indicated under item (ii) of Remark 4. Note that we are now discussing the case $\mu>1$ (an analogous discussion in the $\mu<0$ case can be forsaken), with initial data such that the two circles $B$ and $\Xi$ in the complex $\xi$-plane do intersect and moreover the origin $\xi=0$ falls inside the circle $\Xi$ (i. e. $\left|r_{b}\right|<|\bar{\xi}|+|R|$ and $|\eta|<1$ ).

Let us then consider a given irrational value of $\mu>1$ and let the rational number $\frac{p}{q}$ (with $p>q$ ) provide a very good approximation to $\mu$, which of course entails that the positive integers $p$ and $q$ are both very large. Consider then the difference

$$
\Delta \tilde{j}=\Delta b
$$

(see the second entry in (31) ) between the two positive integers that characterize the two periods of the two time evolutions of $\tilde{w}(t)$ corresponding to two sets of initial data that differ very little. Here clearly the quantity $\Delta b$ is the difference between the number of branch points that are enclosed inside the circle $\Xi$ for these two different sets of initial data. Since the number $q$ of branch points on $B$ is very large, it stands to reason that

$$
\Delta b=\lfloor O(q \delta)\rfloor,
$$

where the quite small (positive) number $\delta$ provides a (dimensionless) measure of the difference between the two sets of initial data (see for instance (43), which clearly 
becomes approximately applicable when $q$ is very large). The floor symbol \lfloor\rfloor has been introduced in the right-hand side of this formula to account for the integer character of the numbers $b$ hence of their difference $\Delta b$, while the order of magnitude symbol $O($ ) indicates that the difference $\Delta b$ is proportional (in fact equal, given the latitude left by our definition of the quantity $\delta$ ) to the quantity $q \delta$ up to corrections which become negligibly small when $\delta$ is very small and $q$ is very large, but irrespective of the value of the quantity $q \delta$ itself which, as the product of the large number $q$ by the small number $\delta$, is required to be neither small nor large.

The relation

$$
\Delta \tilde{j}=\lfloor O(q \delta)\rfloor
$$

implied by this argument indicates that, for any given $q$, one can always choose (finitely different) initial data which differ by such a tiny amount that the corresponding periods are identical, confirming our previous statement about the isochronous character of our model whenever the parameter $\mu$ is rational. But conversely this finding also implies that, for any set of initial data in the sector under present consideration (i. e. that characterized by the inequalities $|\eta|<1$ and $|\bar{\xi}|+|R|>\left|r_{b}\right|$, and by an additional specification to identify the physical root $\breve{w}(t)$, see [4]), if our physical model (11) is characterized by an irrational value of $\mu$, see (17), and one replaces this value by a more and more accurate rational approximation of it, see (20) - as it would for instance be inevitable in any numerical simulation - corresponding to larger and larger values of $p$ and $q$, then one shall have to choose the two different assignments of initial data closer and closer to avoid a drastic change of period - and for these very close sets of data the motion is indeed periodic with a period (which we are able to predict, see (31) and [4], but) which becomes larger and larger the better one approximates the actual, irrational value of $\mu$. Moreover in any numerical simulation the accuracy of the computation, in order to get the correct period, shall also have to increase more and more (with no limit), because of the occurrence of closer and closer near misses through the time evolution (associated with the coming into play of active branch points sitting on the circle $B$ closer and closer to the points of intersection with the circle $\Xi$ ). And finally, if one insists in treating the problem with a truly irrational $\mu$, then, no matter how close the initial data are, the change in the periods becomes infinite because the difference $\Delta b$ in the number of active square-roots branch points on the circle $B$ included inside the circle $\Xi$ is infinite (see (56)), signifying that the motion is aperiodic, and that its evolution is indeed characterized by an infinite number of near misses, making it truly irregular.

This phenomenology, together with that of the near misses as described above, illustrates rather clearly the irregular character of the motions of our physical model when the coupling constants have appropriate values (such as to produce an irrational value of $\mu$ outside the interval $0<\mu<1$ ) and the initial data are in the sector identified above. Note that the Lyapunov coefficients associated with the corresponding trajectories vanish, because these coefficients - as usually defined compare the difference (after an infinitely long time) of two trajectories that, to begin with, differ infinitesimally; whereas our mechanism causing the irregular character of the motion requires, to come into play, an arbitrarily small but finite difference among the initial data. The difference between these two notions corresponds to the fact that inside the interval between two different real numbers - however close they may be there always is an infinity of rational numbers; while this is not the case between two real numbers that differ only infinitesimally! This observation suggests that, in an 
applicative context, the mechanism causing a sensitive dependence on the initial data manifested by our model may be phenomenologically relevant even when no Lyapunov coefficient, defined in the usual manner, is positive. As already observed previously [2], this mechanism is in some sense analogous to that yielding aperiodic trajectories in a triangular billiard with irrational angles; although in that case - in contrast to ours this outcome is mainly attributable to the essentially singular character of the corners, and moreover no truly irregular motions emerge.

\section{Outlook}

In this paper we have introduced and discussed a 3-body problem in the plane suitable to illustrate a mechanism of transition from regular to irregular motions. This model is the simplest one we managed to manufacture for this purpose. Its simplicity permitted us to discuss in considerable detail the mathematical structure underlining this phenomenology: this machinery cannot however be too simple since it must capture (at least some of) the subtleties associated with the onset of an irregular behavior. Therefore in this short paper we were only able to report our main findings without detailing their proofs, and we also omitted several other relevant aspects of our treatment (including a fuller discussion of previous work by others in related areas): this material shall be presented in a separate, much longer, paper [4], and probably as well via an electronic version of our findings so as to supplement their presentation with various animations illustrating these results and their derivation.

Our main motivation to undertake this research project is the hunch that this mechanism of transition have a fairly general validity and be relevant in interesting applicative contexts. Hence we plan to pursue this study by focussing on other cases where this mechanism is known to play a key role, including examples (see, for instance, [2] and [7]) featuring a pattern of branch points covering densely an area of the complex plane of the independent variable rather than being confined just to reside densely on a line as is the case in the model treated herein; and eventually to extend the application of this approach to problems of direct applicative interest.

In this connection the following final observation is perhaps relevant. In this paper as well as in others $[1,2,7]$ the main focus has been on models featuring a transition from an isochronous to an irregular regime, and in this context much emphasis was put on the "trick" (7) and in particular on the relationship it entails between the periodicity of the ("physical") dependent variables $z_{n}(t)$ as functions of the real independent variable $t$ ("time") and the analyticity of other, related ("auxiliary") dependent variables $\zeta_{n}(\tau)$ as functions of a complex independent variable $\tau$. But our findings can also be interpreted directly in terms of the analytic properties of the physical dependent variables $z_{n}(t)$ as functions of the independent variable $t$ considered itself as a complex variable. Then the time evolution, which corresponded to a uniform travel round and round on the circle $C$ in the complex $\tau$-plane or equivalently on the circle $\Xi$ in the complex $\xi$-plane, is represented as a uniform travel to the right along the real axis in the complex $t$-plane, while, via the relations (see (7) and (15))

$$
t=(2 \mathrm{i} \omega)^{-1} \log (1+2 \mathrm{i} \omega \tau)=(2 \mathrm{i} \omega)^{-1} \log \left(\frac{\xi-\bar{\xi}}{R}\right),
$$

the pattern of branch points in the complex $\tau$-plane or equivalently in the complex $\xi$ plane gets mapped into a somewhat analogous pattern in the complex $t$-plane, repeated 
periodically in the real direction with period $T$, see (2). In particular - to mention the main features relevant to our treatment, see above - the circle $B$ on which the square-root branch points in the complex $\xi$-plane sit, gets mapped in the complex $t$ plane into a curve $\hat{B}$ on which sit the square-root branch points in the complex $t$-plane; note that this curve $\hat{B}$ (in contrast to the circle $B$ ) does now depend on the initial data. This curve is of course repeated periodically; it is closed and contained in each vertical slab of width $T$ (see figure 2 $\mathrm{b}$ ) if the point $\bar{\xi}$ is outside $B$, otherwise it is open, starting in one slab and ending in the adjoining slab at a point shifted by the amount $T$; and it does not or does cross (of course twice in each period) the real axis in the complex $t$-plane depending whether, in the complex $\xi$-plane, the two circles $B$ and $\Xi$ do not or do intersect each other (see figure 2 k). Likewise, depending whether it is
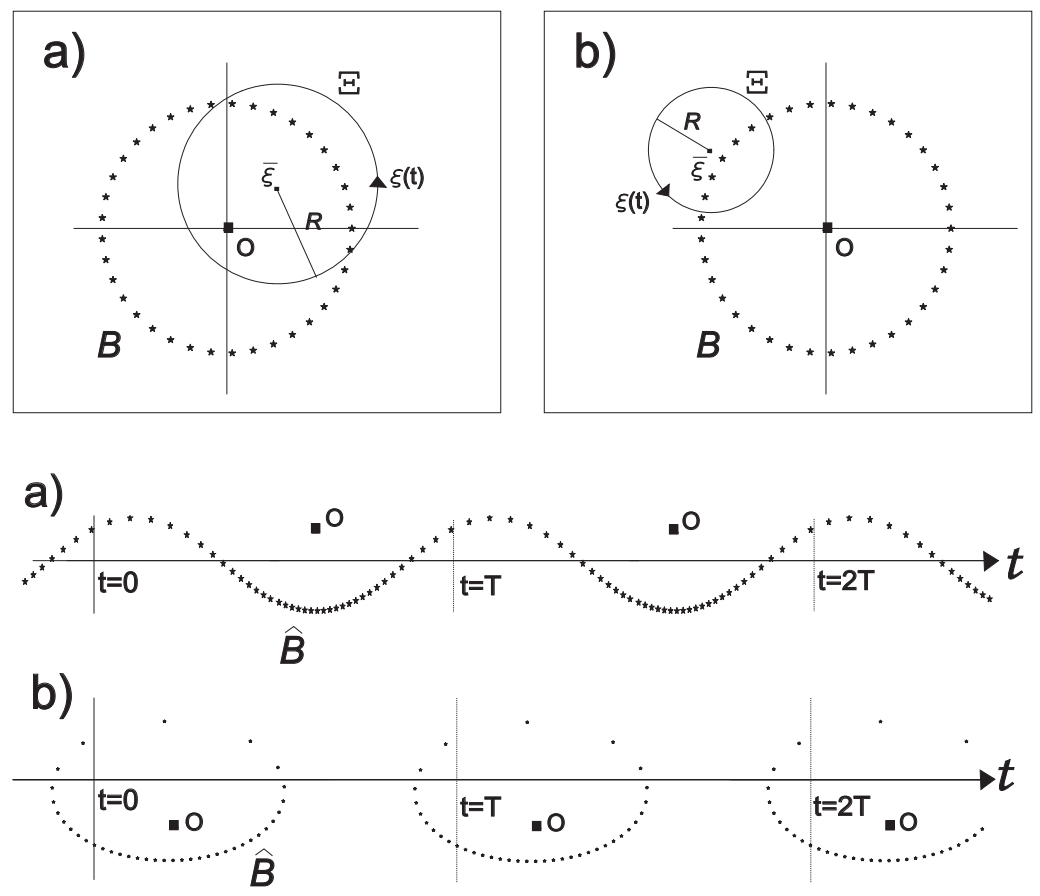

Figure 2. Evolutionary paths and branch points of the solutions in the complex $\xi$-plane and $t$-plane for $\mu=15 / 37$.

inside or outside the circle $\Xi$, the point $\xi=0$ - which, as entailed by our analysis, is a highly relevant branch point in the complex $\xi$-plane (unless $0<\mu<1$ ) - gets mapped into an analogous branch point located in each vertical slab above or below the real axis in the complex $t$-plane; while the other branch point, at $\xi=\infty$ in the complex $\xi$-plane, gets mapped into an analogous branch point located at infinity in the lower half of the complex $t$-plane. Clearly the physical mechanism of near misses, which is the main cause of the eventual irregularity of the motion, becomes relevant only for initial data such that the curve $\hat{B}$ crosses the real axis, thereby causing (if $\mu$ is irrational) an infinity of square-root branch points of the functions $z_{n}(t)$ to occur arbitrarily close to the real axis in the complex t-plane - branch points which are however active (namely, they actually cause a near miss in the physical evolution) in only some (yet still an infinity) of the infinite number of vertical slabs in which 
the complex t-plane gets now naturally partitioned. The near miss implies that the two particles involved in it slide past each other from one side or the other depending whether the corresponding branch point is just above or just below the real axis in the complex $t$-plane. The sensitive dependence on the initial data is due to the fact that any tiny change of them causes some active branch point in the complex $t$-plane which is very close to real axis to cross over from one side of it to the other, thereby drastically changing the outcome of the corresponding near miss.

This terse discussion shows clearly that the explanation of the irregular behavior of a dynamical system in terms of travel on a Riemann surface is by no means restricted to isochronous systems. We found it convenient to illustrate in detail this paradigm by focussing in this paper on a simple isochronous model and by using firstly $\tau$ and then $\xi$ as independent complex variables - but, as outlined just above, our analysis can also be done - albeit less neatly - by using directly the independent complex variable $t$; and the occurrence of a kind of periodic partition of the complex $t$-plane into an infinite sequence of vertical slabs - characteristic of our isochronous model - does not play an essential role to explain the irregular character of the motion when such a phenomenology does indeed emerge. The essential point is the possibility to reinterpret the time evolution as travel on a Riemann surface, the structure of which is sufficiently complicated to cause an irregular motion featuring a sensitive dependence on its initial data. The essential feature causing such an outcome is the presence of an infinity of branch points arbitrarily close to the real axis in the complex $t$-plane, the positions of which, as well as the identification of which of them are active, depends on the initial data nontrivially. The model treated in this paper shows that such a structure can be complicated enough to cause an irregular motion, yet amenable to a simple mathematical description yielding a rather detailed understanding of this motion; this suggests the efficacy also in more general contexts of this paradigm to understand (certain) irregular motions featuring a sensitive dependence on their initial data and possibly even to predict their behavior to the extent such a paradoxical achievement (predicting the unpredictable!) can at all be feasible.

\section{Acknowledgments}

It is a pleasure to acknowledge illuminating discussions with Boris Dubrovin, Yuri Fedorov, Jean-Pierre Françoise, François Leyvraz, Jaume Llibre, Alexander Mikhailov and Carles Simó. 
[1] F. Calogero, A class of integrable Hamiltonian systems whose solutions are (perhaps) all completely periodic, J. Math. Phys. 38, 5711-5719 (1997); Differential equations featuring many periodic solutions, in: L. Mason and Y. Nutku (eds), Geometry and integrability, London Mathematical Society Lecture Notes, vol. 295, Cambridge University Press, Cambridge, 2003, pp. 9-21; Periodic solutions of a system of complex ODEs, Phys. Lett. A293, 146-150 (2002); On a modified version of a solvable ODE due to Painlevé, J. Phys. A: Math. Gen. 35, 985992 (2002); On modified versions of some solvable ODEs due to Chazy, J. Phys. A: Math. Gen. 35, 4249-4256 (2002); Solvable three-body problem and Painlevé conjectures, Theor. Math. Phys. 133, 1443-1452 (2002); Erratum 134, 139 (2003); A complex deformation of the classical gravitational many-body problem that features a lot of completely periodic motions, J. Phys. A: Math. Gen. 35, 3619-3627 (2002); Partially superintegrable (indeed isochronous) systems are not rare, in: New Trends in Integrability and Partial Solvability, edited by A. B. Shabat, A. Gonzalez-Lopez, M. Mañas, L. Martinez Alonso and M. A. Rodriguez, NATO Science Series, II. Mathematics, Physics and Chemistry, vol. 132, Proceedings of the NATO Advanced Research Workshop held in Cadiz, Spain, 2-16 June 2002, Kluwer, 2004, pp. 49-77; General solution of a three-body problem in the plane, J. Phys. A: Math. Gen. 36, 7291-7299 (2003); Solution of the goldfish N-body problem in the plane with (only) nearest-neighbor coupling constants all equal to minus one half, J. Nonlinear Math. Phys.11, 1-11 (2004); Two new classes of isochronous Hamiltonian systems, J. Nonlinear Math. Phys. 11, 208-222 (2004); Isochronous dynamical systems, Applicable Anal. (in press); A technique to identify solvable dynamical systems, and a solvable generalization of the goldfish many-body problem, J. Math. Phys. 45, 2266-2279 (2004); A technique to identify solvable dynamical systems, and another solvable extension of the goldfish many-body problem, J. Math. Phys. 45, 4661-4678 (2004); Isochronous systems, Proceedings of the Conference on Geometry, Integrability and Physics, Varna, June 2004 (in press); Isochronous systems, in: Encyclopedia of Mathematical Physics, edited by J.-P Françoise, G. Naber and Tsou Sheung Tsun (in press). F. Calogero and A. Degasperis, Novel solution of the integrable system describing the resonant interaction of three waves, Physica D 200, 242-256 (2005). F. Calogero and J.-P. Françoise, Periodic solutions of a many-rotator problem in the plane, Inverse Problems 17, 1-8 (2001); Periodic motions galore: how to modify nonlinear evolution equations so that they feature a lot of periodic solutions, J. Nonlinear Math. Phys. 9, 99-125 (2002); Nonlinear evolution ODEs featuring many periodic solutions, Theor. Math. Phys. 137, 1663-1675 (2003); Isochronous motions galore: nonlinearly coupled oscillators with lots of isochronous solutions, in: Superintegrability in Classical and Quantum Systems, Proceedings of the Workshop on Superintegrability in Classical and Quantum Systems, Centre de Recherches Mathématiques (CRM), Université de Montréal, September 16-21 (2003), CRM Proceedings \& Lecture Notes, vol. 37, American Mathematical Society, 2004, pp. 15-27; New solvable many-body problems in the plane, Annales Henri Poincaré (submitted to). F. Calogero, J.-P. Françoise and A. Guillot, A further solvable three-body problem in the plane, J. Math. Phys. 10, 157-214 (2003). F. Calogero and V. I. Inozemtsev, Nonlinear harmonic oscillators, J. Phys. A: Math. Gen. 35, 1036510375 (2002). S. Iona and F. Calogero, Integrable systems of quartic oscillators in ordinary (three-dimensional) space, J. Phys. A: Math. Gen. 35, 3091-3098 (2002). M. Mariani and F. Calogero, "Isochronous PDEs", Yadernaya Fizika (Russian Journal of Nuclear Physics) 68, 958-968 (2005); A modified Schwarzian Korteweg de Vries equation in 2+1 dimensions with lots of periodic solutions, Yadernaya Fizika(in press). M. Sommacal, "Studio di problemi a molti corpi nel piano con tecniche numeriche ed analitiche", Dissertaion for the "Laurea in Fisica", Università di Roma "La Sapienza", 26 September 2002.

[2] F. Calogero and M. Sommacal, Periodic solutions of a system of complex ODEs. II. Higher periods, J. Nonlinear Math. Phys. 9, 1-33 (2002). F. Calogero, J.-P. Françoise and M. Sommacal, Periodic solutions of a many-rotator problem in the plane. II. Analysis of various motions, $J$. Nonlinear Math. Phys. 10, 157-214 (2003).

[3] F. Calogero, Classical Many-Body Problems Amenable to Exact Treatments, Lecture Notes in Physics Monograph m66, Springer, Berlin, 2001.

[4] F. Calogero, D. Gomez-Ullate, P. M. Santini and M. Sommacal, The transition from regular to irregular motion explained as travel on Riemann surfaces. II (to be published).

[5] M. D. Kruskal and P. A. Clarkson, The Painlevé-Kowalewski and poly-Painlevé tests for integrability, Studies Appl. Math. 86, 87-165 (1992). R. D. Costin and M. D. Kruskal, Nonintegrability criteria for a class of differential equations with two regular singular points, Nonlinearity 16, 1295-1317 (2003). R. D. Costin, Integrability properties of a generalized Lamé equation; applications to the Hénon-Heiles system, Methods Appl. Anal. 4, 113-123 (1997).

[6] T. Bountis, L. Drossos and I. C. Percival, Non-integrable systems with algebraic singularities 
in complex time, J. Phys. A: Math. Gen. 23, 3217-3236 (1991). T. Bountis, Investigating non-integrability and chaos in complex time, in: NATO ASI Conf. Proc., Como, September 1993 \& Phys. D 86, 256-267 (1995); Investigating non-integrability and chaos in complex time, Physica D86, 256-267 (1995). A. S. Fokas and T. Bountis, Order and the ubiquitous occurrence of chaos, Physica A228, 236-244 (1996).S. Abenda, V. Marinakis and T. Bountis, On the connection between hyperelliptic separability and Painlevé integrability, J. Phys. A: Math. Gen. 34, 3521-3539 (2001).

[7] E. Induti, "Studio del moto nel piano complesso di $N$ particelle attirate verso l' origine da una forza lineare ed interagenti a coppie con una forza proporzionale ad una potenza inversa dispari della loro mutua distanza", Dissertation for the "Laurea in Fisica", Università di Roma "La Sapienza", 26 May 2005. 